\title{
AI Enabled Self Diagnosis Predictor Tool using Tongue Image Capture with Automatic Prescription Generation
}

\author{
Dhwani Trivedi, Bhargav Goradiya, Ghanshyam Rathod
}

\begin{abstract}
WHO data shows that half of the people in the world suffer due to basic health care needs as there are not enough medical facilities available in many parts of the world. It is difficult for the refugees to have all the basic health care needs and not enough doctors available for primary diagnosis. To diagnose the person there are many methods by which the doctor can predict what type disease one might be suffering from. One of those factors includes the first diagnosis done by just observing the tongue, as it's the only visible part of the body and one of the factors which helps for primary diagnosis and widely accepted by doctors in TCM, diagnosis. It addresses for an aid to people to do primary diagnosis from tongue using AI device, like Raspberry Pi with camera, which is trained using tongue dataset of different types of tongue images like strawberry tongue, Black tongue, normal tongue, Red tongue, Swallowed tongue etc. for various symptoms of various diseases to identify the type of the tongue and based on that it will generate the prescription. The proposed research work is based on the edge computing and does not need any internet or cloud support and best suitable for installing as portable kiosk in affected areas where primary medical facility is not available. The report generated by system has primary predicted suggestions based on the tongue diagnosis using AI.
\end{abstract}

Keywords: ICT in Health, Artificial Intelligence, Primary Diagnosis, Health prediction, Edge Computing

\section{INTRODUCTION}

To diagnose the health of a person there are lots of factors through which the doctor can predict what the disease might one be suffering from. One of those factors include the diagnosis done by just looking at the tongue as it's the only visible part of the digestive system and hence can predict the overall health of the body as well. The tongue describes overall health of the body system and metabolic and nutritive health of a person. Change in tongue's texture, color, shape, size can predict the disease one might be suffering from and also predict which body part might be affected due to it. The Chinese medicine, Ayurveda and Greek physicians also have considered the tongue as a important predictor for diagnosing the body at first instance. These days thermometers are found in every home just to measure the temperature to primarily understand the body temperature and helps to do self-diagnosis at primary level, The same way if an AI enabled system can help us to understand the human tongue type, one

Revised Manuscript Received on May 20, 2020.

* Correspondence Author

Dhwani Trivedi*, EC, BVM, Engineering College, Anand, India. Email dhwanitrivedi999@gmail.com

Dr. Bhargav Goradiya EC, BVM, Engineering College, Anand, India Email: bhargav.goradiya@bvmengineering.ac.in

Dr. Ghanshyam Rathod, EC, BVM, Engineering College, Anand, India Email: ghansyam.rathod@bvmengineering.ac.in can easily do self-diagnosis which can help a person to visit doctors in time before the dieses grows much. There are places in the world where people do not have enough medical facility to monitor their health as there are no doctors available. Hence, to overcome that difficulty an AI enabled health predictor system can be made so that just by clicking the picture of the tongue the machine will be able to predict through transfer learning method of machine learning, what sort of disease a person might be suffering from and might suggest to consult a doctor for further diagnosis. The system does claim about specific diseases but surely gives a list of possible health problems one might be suffering from giving details about, the type of the tongue and based on that it will generate an automatic prescription which contains the accuracy, type of tongue and predicted possible health problem, later it will be easier for the doctor and the patient for diagnosing the disease it will also helpful for the doctor in making the diagnosis process easier as it is generated by a trained AI model using thousands of data.

\section{METHODOLOGY}

There are various tongue related ways to identify the disease a person is suffering from. The tongue is supposed to be the starting point for diagnosing the patient's health. The prediction is based on various factors which includes the color, textures, and the surface of the tongue. There are various types of tongue except from the anomaly tongue like based on the textures are the Geographical Tongue, Fissure Tongue based on colors there is Yellow, tongue, Red or Angry tongue, Purple tongue, Magenta Black or Hairy Tongue. Depending on the color texture and features of the tongue the prediction is done on the basis of the same. Also, the tongue is considered as a map according to the Chinese medicine which describes that the tip of the tongue is connected to the, the back is connected to the kidney the sides to the liver. Depending upon the different types of tongue the person's health can be predicted. If the person has red spot on the side of the tongue it might result a person suffering from cancer or HIV, red color suggests that a person might be suffering from acute fever, magenta color describes the deficiency of riboflavin, strawberry tongue represents a scarlet fever or an acute fever hence just a looking a patients tongue the doctor is able to predict what sort of disease a person is suffering from hence the same concept here is applied to train the machine in a similar way

Published By:

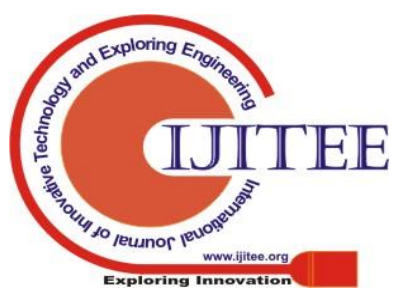




\section{A. Dataset Preparation}

As in for the prototype version of the system the samples of tongue have been obtained varying from color, texture, shape and size. They have been labelled as fissured tongue, hairy tongue, normal tongue, HIV etc. and sorted out the images accordingly. After classifying manually, the types of tongue were given as labels. Improper and blur images were removed. Different images of tongues were kept in different folders as labels as shown in figure 1

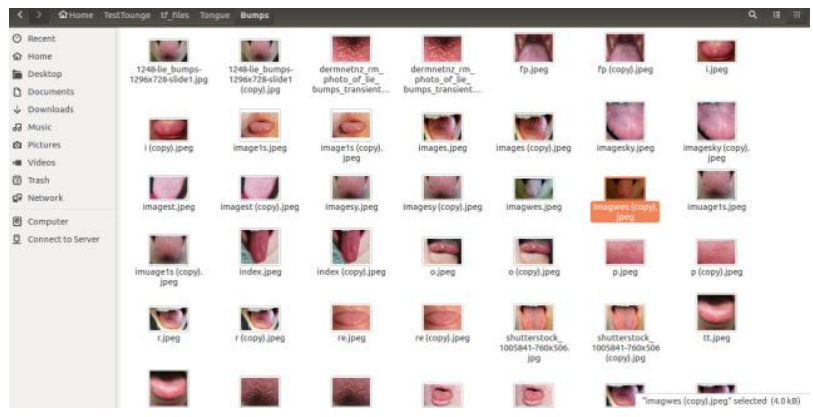

Now the images are kept in the respective folder for their purposes, which are supposed to be done through the Raspberry Pi shown in figure Now the dataset is ready for being trained by the Inception model as shown in figure 5 using transfer learning, which is the pre-trained model in which the last layer of the $\mathrm{CNN}$ has been trained and due to that the model is trained by the concept of Transfer Learning using Raspberry $\mathrm{Pi}$ and deployed. Here are some of the samples of various types of tongues based on color, texture, shape and size as shown in figure 2, 3 and 4

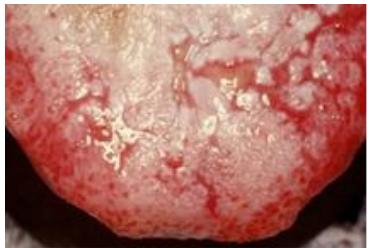

Figure 2: Geographical Tongue

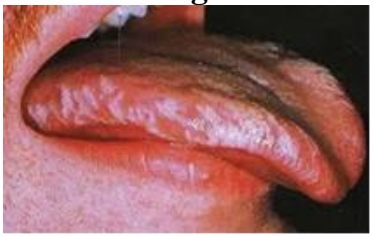

Figure 4: HIV Infected Tongue

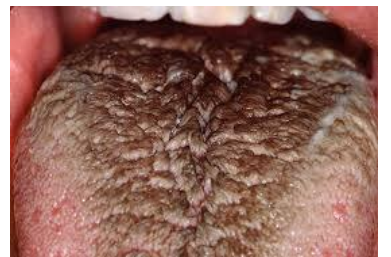

Figure 3: Hairy Tongue

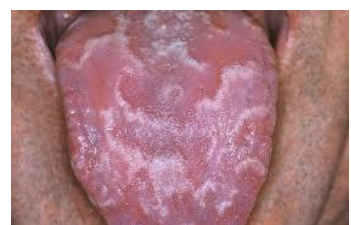

Figure 5: Geographical Tongue

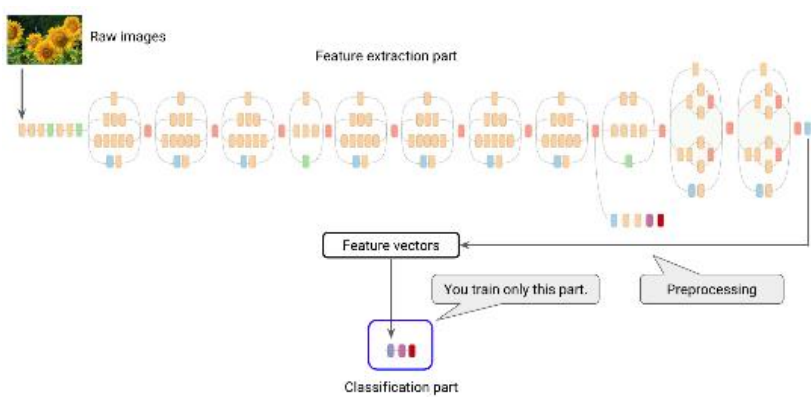

Figure 5 : Inception Architecture Source: Google code lab Now the model obtained after the dataset which has been trained properly and is ready for testing for a Health Prediction system using the Raspberry Pi3
B. Setting up Raspberry Pi 3 and making an AI model using TF Lite
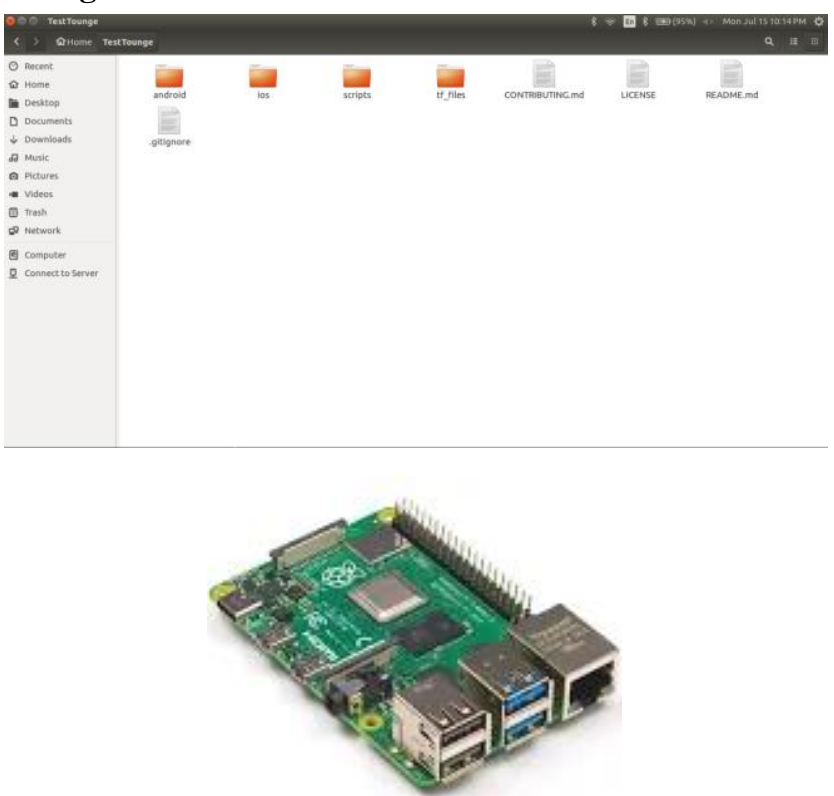

Figure 5 and Figure 6 : Dataset and Raspberry Pi

The bottlenecks that will be created are shown in the below figure, which represents the number of counts of the layers that have been trained for the model. The greater number of bottlenecks created more number of layers are present hence the model has been trained accurately.

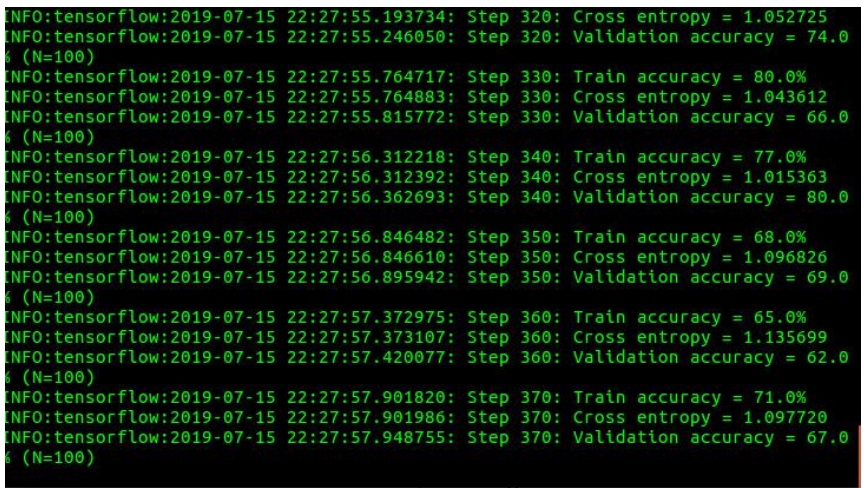

Figure 7: Re training of the inception model

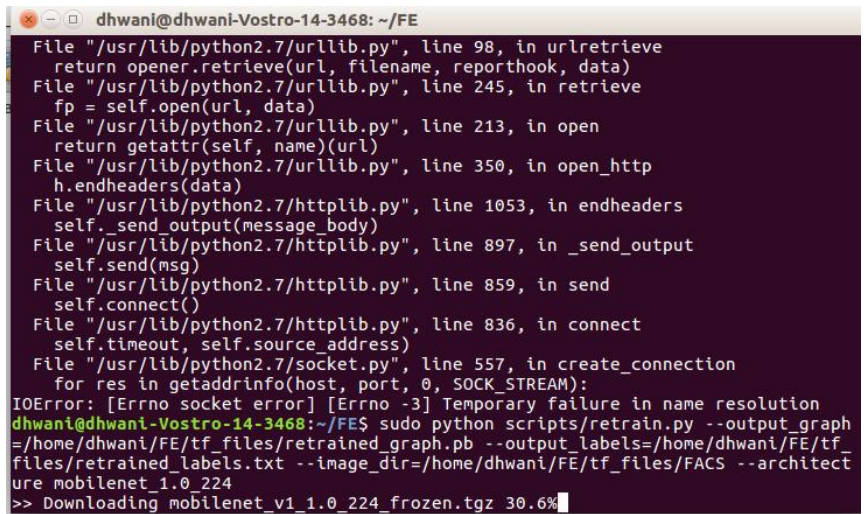

Figure 8: Downloading the Mobilenet Architecture

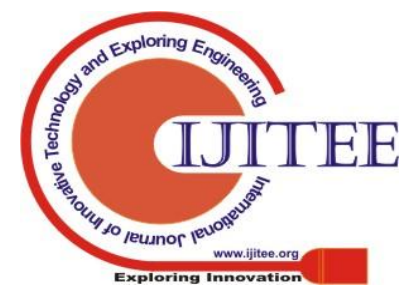


To see the training and the testing accuracy it is shown in the below table.

Table 1: Testing and Train Accuracy

\begin{tabular}{|c|c|c|}
\hline Steps & $\begin{array}{c}\text { Train Accuracy } \\
(\%)\end{array}$ & $\begin{array}{c}\text { Test Accuracy } \\
(\%)\end{array}$ \\
\hline 1 & $34 \%$ & $20 \%$ \\
\hline 2 & $67 \%$ & $50 \%$ \\
\hline 3 & $94.12 \%$ & $95 \%$ \\
\hline
\end{tabular}

When the model will be trained, there will be two files that will be created in the Tongue folder known as retrained_graph.pb and retrained_label.txt These two files represent the values that has been obtained after retraining the model through our model and the one which will have names of the labels that we have labelled accordingly. The size of the retrained_graph. pb is $171 \mathrm{MB}$.

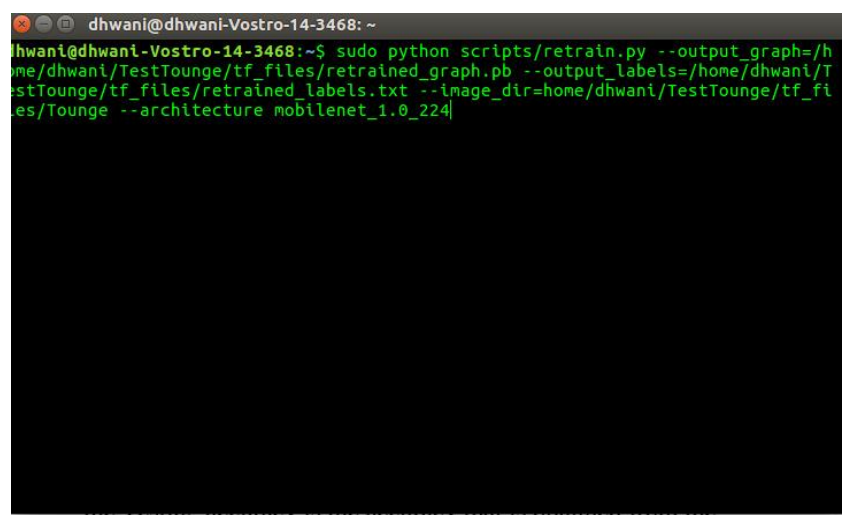

Figure 9: Generation of retrained_graph.pb and retrained_labels.txt

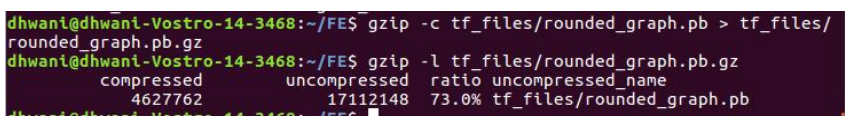

Figure 10: Compressing the retrained file obtained to the optimized file.

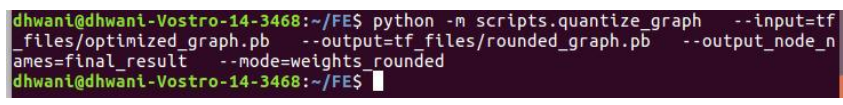

Figure 11: Conversion of retrained_graph. pb to rounded_graph.pb (optimized_graph.pb)

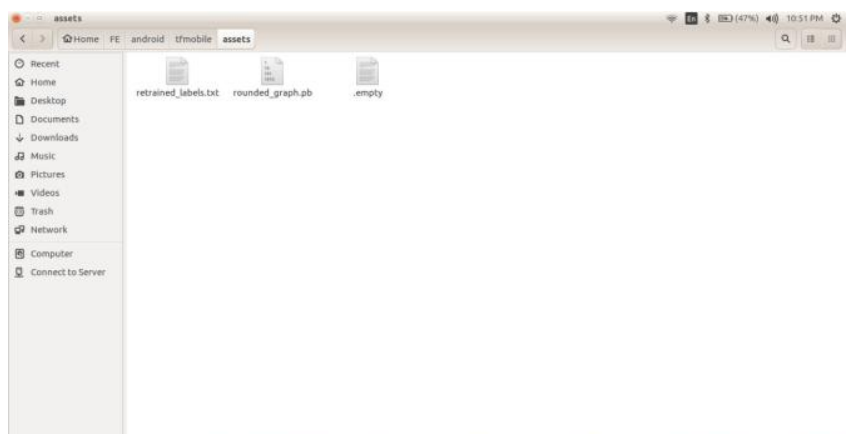

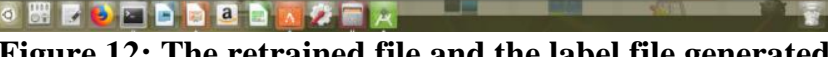

Figure 12: The retrained file and the label file generated in the tf_files folder.

\section{GENERATION OF PRESCRIPTION}

Using PyPDF python library to generate pdf file, the automatic prescription is generated from Raspberry Pi and sent to Telegram messenger application. The pdf file contains the photo of the tongue under diagnosis and symptoms are added as labels in the file. The screenshot of the telegram is shown in the figure 13
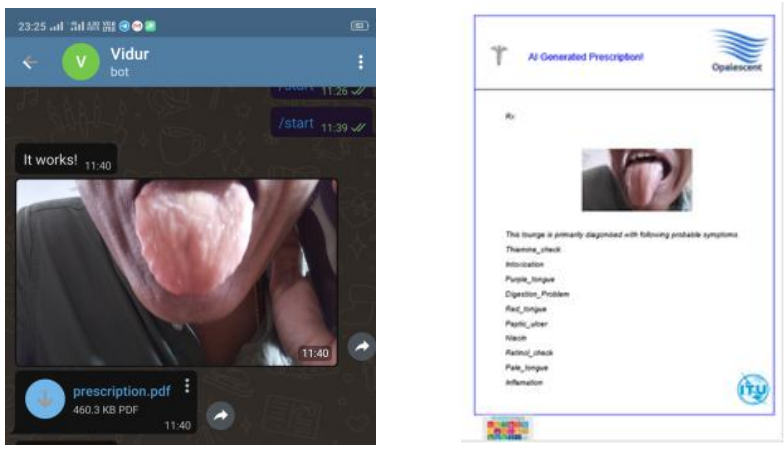

Figure 13: Telegram messenger and autogenerated pdf

\section{FUTURE SCOPE}

This system can be useful for the people that do not have enough facilities especially in underdeveloped countries where are there no doctors available for the primary diagnosis using this app the patient will be able to predict what disease he might be suffering from and as the app does not require any internet connectivity hence it will be really helpful. Technology Aided healthcare device might be a boon for both the doctors and the people who do not have enough facility for basic health treatment and AI might be helpful in saving lots of lives.

\section{REFERENCES}

1. Tongue

diagnoses -https://www.nhp.gov.in/uploadfiles/microsite/635846561062513669 _1.pdf

2. TensorFlow for poets for developing the model on

3. Raspberry $\mathrm{Pi}$ hardware https://codelabs.developers.google.com/codelabs/tensorflow-for-poets $-2$

4. TensorFlow for Poets 2: TFLite Android

5. https://codelabs.developers.google.com/codelabs/tensorflow-for-poets -2-tflite/\#0

6. 4 Google's Inception model V3.

7. https://www.tensorflow.org/tutorials/images/hub_with_keras

8. NVIDIA Jetson Nano https://developer.nvidia.com/embedded/jetson-nano-developer-kit

9. L.Yao et al., "Discovering treatment pattern in traditional Chinese medicine clinical cases by exploiting supervised topic model and domain knowledge,” J. Biomed. Informant., vol. 58, pp. 260-267, Dec. 2015 .

10. A. Kumar, J. Kim, D. Lyndon, M. Fulham, and D. Feng, "An ensemble of fine-tuned convolutional neural networks for medical image classification,” IEEE J. Biomed. Health Informant. vol. 21, no. 1, pp. 31-40, Jan. 2017.

11. S. S. Girija. (2016). TensorFlow: Large-Scale Machine Learning on Heterogeneous Distributed Systems. [Online]. Available: https://www.tensorflow.org/

12. K. Simonyan and A. Zisserman, "Very deep convolutional networks for large-scale image recognition," arXiv preprint arXiv: 1409.1556, 2014.

\section{AUTHORS PROFILE}

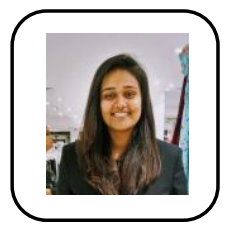

Dhwani Trivedi: Dhwani Trivedi is an Electronics \& Communication Engineering student from BVM Engineering College, Anand affiliated with Gujarat Technological University, Gujarat, India. 
She loves to be part of Maker activities and keeping herself pace with cutting age AI tools. She is currently involved in learning AI and related innovations. She is keen to contribute to this changing world by her efforts on AI for connected world. She had been invited at UNESCO Paris HQ during Mobile Learning Week for her symposium. She had participated in LoRa Workshop at ICTP, Italy. Dhwani Trivedi had published few posters and papers on AI.

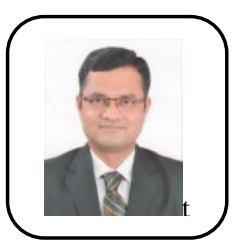

Dr. Bhargav Goradiya Dr. Bhargav Goradiya is seasoned academician, having more than two decades of experience. Presently Dr. Goradiya is professor and head of electronics and communication engineering department at BVM engineering college. He is having keen interest of developing sustainable, affordable, low cost and robust solutions for smart cities. His area of interest is towards Machine learning, IoT and Medical electronics. He was instrumental in fetching world bank supported MHRD TEQIP II grant of rupees 10 crore at BVM. Dr. Goradiya was Pioneer in promoting the entrepreneurship and has established BVM incubation center for promoting student startup activities. He is having strong belief that engineering education is not within four walls of classroom but in constant and continuous interaction with industries. He has setup center of excellence in the domain of IoT infrastructure in association with System Level Solution (SLS) at BVM.

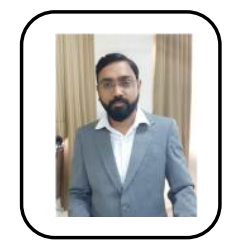

Dr. Ghansyam Rathod: Dr. Ghansyam Rathod is currently working as an Assistant Professor in Electronics and Communication engineering department of Birla Vishwakarma Mahavidya. He is having teaching experience more than a decade in the field of Electronics and communication. His research areas of interest are Biomedical Instrumentation, Signal Processing, Analog Communication etc. He has guided more than 20 under graduate projects as well published more than 10 research papers in Journals and conferences. He is also active member of various core committee of institutions. He has a subject experience of Biomedical Instrumentation, Network theory, Electromagnetic Engineering, Electronics Measurements and Instrumentation, Basic Electronics, Control system etc. $\mathrm{He}$ is done UG in Electronics Engineering, Masters in Communication engineering and right now pursuing part time $\mathrm{PhD}$ in Biomedical Signal Processing. 